\title{
Lacunarity as a Texture Measure for Address Block Segmentation
}

\author{
Jacques Facon $^{1}$, David Menoti ${ }^{1,2}$, and Arnaldo de Albuquerque Araújo ${ }^{2}$ \\ 1 PUCPR - Pontifícia Universidade Católica do Paraná, \\ Grupo de Imagem e Visão - Programa de Pós-Graduação em Informática Aplicada, \\ Rua Imaculada Conceição, 1155, Prado Velho - 80.215-901, Curitiba-PR, Brazil \\ ffacon, menoti\}@ppgia.pucpr.br \\ 2 UFMG - Universidade Federal de Minas Gerais, \\ Grupo de Processamento Digital de Imagens - Departamento de Ciência da Computação, \\ Av. Antônio Carlos, 6627, Pampulha - 31.270-010, Belo Horizonte-MG, Brazil \\ \{menoti, arnaldo\}@dcc.ufmg.br
}

\begin{abstract}
In this paper, an approach based on lacunarity to locate address blocks in postal envelopes is proposed. After computing the lacunarity of a postal envelope image, a non-linear transformation is applied on it. A thresholding technique is then used to generate evidences. Finally, a region growing is applied to reconstruct semantic objects like stamps, postmarks, and address blocks. Very little $a$ priori knowledge of the envelope images is required. By using the lacunarity for several ranges of neighbor window sizes $r$ onto 200 postal envelope images, the proposed approach reached a success rate over than $97 \%$ on average.
\end{abstract}

\section{Introduction}

Postal Service processes postal envelopes through manual and automated operations. The former require an employee to read the address before sorting the mail. The latter requires that an employee simply feed mail into and remove mail from a machine that both "reads" and sorts. Due to wide variety of postal envelope attributes like layouts, colors, texture, and handwritten address block mixed up with postmarks or stamps, many mails have to be processed manually. Mail-handling is a very labor intensive process and the knowledge level required for the sorting process is quite considerable. The use of automation is the logical choice for improving productivity and reducing expenses. Mail sorting and postal automation represent an important area of application for Image Processing and Pattern Recognition techniques. The main function required in postal automation, involving Computer Vision, is definitely address reading and interpretation.

Here, we have focused our attention on segmentation of a postal envelope image into stamps, postmarks and address blocks. Other works in the literature have tackled different aspects of that problem. An address block location method is proposed in [1] for working with both machine and hand printed addresses. The method is based on dividing the input image into blocks where the homogeneity of each block gradient magnitude is measured. Heuristically given thresholds are used to decide upon the gradient magnitude of a typical address block candidate. In their tests 1600 machine 
printed addresses and 400 hand printed ones were used, reporting over $91 \%$ successful location. The solution appears to work fast for well-constrained envelopes, whereby a large separation exists between the image regions since they mentioned a large drawback in the figures if the envelopes have more than one stamp for example. Eiterer et al [2] present a segmentation method based on calculation of fractal dimension from $2 \mathrm{D}$ variation procedure and k-means clustering. The authors have also computed the influence of box size $r$ used in each image pixel. Best values, on a 200 postal envelope database, were obtained for range $r=\{3,9\}$, where the segmentation recovered address blocks $(97.24 \% \pm 13.64 \%)$ with quite noise $(6.43 \% \pm 6.52 \%)$.

The purpose of this study is to investigate the potential usefulness of lacunarity in quantifying the texture of postal envelope images to locate handwritten address blocks, postmarks and stamps, with little a priori knowledge of the envelope images.

The rest of this paper is organized as follows: Section 2 describes the segmentation task for postal automation and the proposed approach. Section 3 presents some experimental results, the evaluation process used and briefly a discussion. Finally, some conclusions are drawn in Section 4

\section{The Proposed Segmentation Approach for Postal Automation}

The segmentation task to be performed for postal automation consists in separating the background, and locating the address block, stamps, and postmarks. Our postal envelope segmentation approach is based on evidence generation by lacunarity associated with a region-growing algorithm. The 4 main steps are (Figure1):

- Feature Extraction: it is performed on an input image $I_{i n}$ by means of Lacunarity generating a feature image $I_{F E}$;

- Feature Normalization: New features $I_{F N}$ are devised by non-linear normalization from $I_{F E}$, in order to enhance singularities (discontinuities) between background and objects and enhance extracted features;

- Saliency Identification: it is performed from $I_{F N}$ by a thresholding algorithm generating $I_{S I}$, which contains enough evidence for segmentation objects;

- Region-growing: it is applied on the evidences in $I_{S I}$ in order to recover all remaining pixels belonging to segmentation objects of interest, yelding the final segmentation $I_{\text {out }}$.

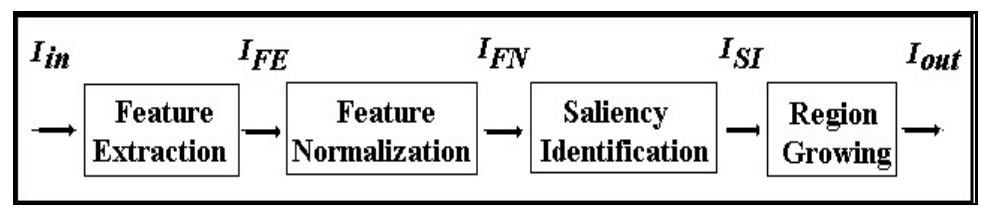

Fig. 1. Flowchart of the segmentation approach proposed 


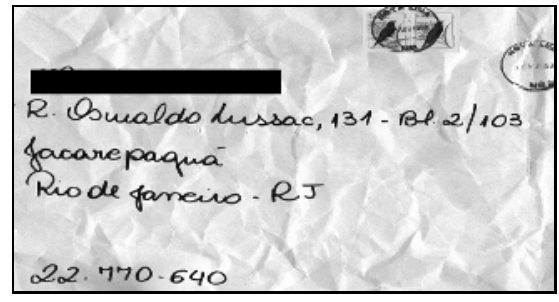

(a)

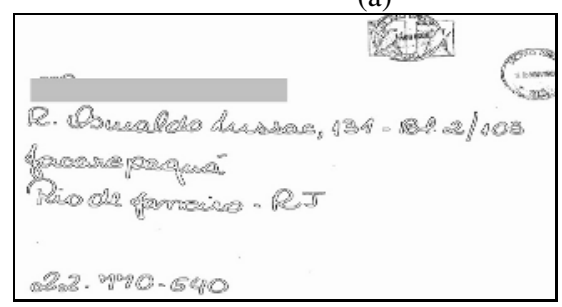

(c)

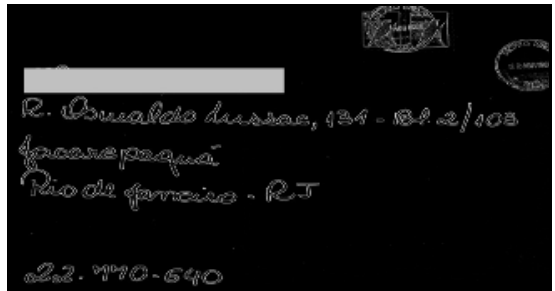

(b)

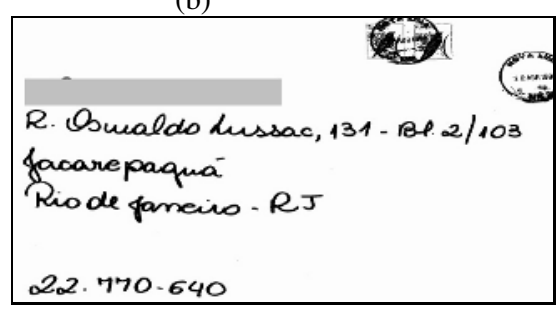

(d)

Fig. 2. Input image and outputs of the last three steps: (a) Envelope image, (b) Feature Normalization, (c) Saliency Identification, (d) Region Growing

\subsection{Feature Extraction}

Lacunarity is a multi-scale measure describing the distribution of gaps within a texture: the greater the range in gap size distribution, the more lacunar the data [3]. Higher lacunarity values represent a wider range of sizes of structures within an image. Lacunarity is sensitive to both the image density and its spatial configuration [4].

A number of algorithms have been proposed for measuring this property [5], [6]. The Allain 's and Cloitre 's [7] algorithm for lacunarity estimation gliding box method has been adopted. The gliding-box samples an image using overlapping square windows of length $r$. Lacunarity is defined in terms of the local first and second moments, measured for each neighborhood size, about every pixel in the image [4]:

$$
L(r)=1+\frac{\operatorname{var}(r)}{\operatorname{mean}^{2}(r)}
$$

where mean $(r)$ and $\operatorname{var}(r)$ are the mean and variance of the pixel values, respectively, for a neighborhood size $r$.

Thus, lacunarity is used as evidence to distinguish between background and objects. Feature extraction $\left(I_{F E}\right)$ is performed by using Equation 1 , where mean $(r)$ and $\operatorname{var}(r)$ will be computed for different neighborhood sizes $r$.

\subsection{Feature Normalization}

The distribution in $I_{F E}$ is very sparse and non-uniform. How to detect the lacunarity values that can capture texture characteristic for homogeneous areas or for transition areas is the main challenge for this feature-developing task. The variations of handwritten object sizes and background illumination in image directly affect lacunarity 
distribution. To take into account the variation of lacunarity distributions, a non-linear normalization was used:

$$
I_{F N}=\arctan \left(\frac{I_{F E}}{\left(k * s t d\left(I_{F E}\right)\right.}\right)
$$

where $\arctan (\bullet)$ is a trigonometric and well-known non-linear function, $\operatorname{std}\left(I_{F E}\right)$ is standard deviation of $I_{F E}$ and $k$ is a multiplicative factor. Figure 2-(b) depicts an example where it is easy to observe the enhancement of evidences.

\subsection{Saliency Identification}

To separate evidences into objects and background, the Otsu 's thresholding algorithm [8] was used, producing the output $I_{S I}$ (Figure2-(c)).

Once features devised in the first two steps are thresholded, saliencies for segmentation objects are detected. Thus, they are working as evidences for next step to reconstruct desired objects. Figure2-(d) depicts this step.

\subsection{Region Growing}

At this stage, the image $I_{S I}$ contains the selected evidences likely to belong to either address block, stamps or postmarks. However, these evidences have to be properly used in order to select the coherent pixels for $I_{S I}$.

Thus, each point in $I_{S I}$ will be selected if the gray value, $i_{v}$, of respective pixel falls inside $\lambda \%$ of image distribution (Gaussian):

$$
i_{v} \leq I_{\mu}-Z_{50 \%-\lambda} \times I_{\sigma}
$$

where, $I_{\mu}$ and $I_{\sigma}$ are the global mean and global standard deviation of image, respectively, $Z_{50 \%-\lambda}$ is the normalized point for probability of $50 \%-\lambda$. In fact, we suppose that objects to be recovered are the $\lambda \%$ (in Gaussian distribution) darker ones.

After verifying all points $i_{v}$ indicated through $I_{S I}$, only ones that hold the global constrains (Equation 3) will be stored:

$$
i_{v} \leq i_{g}
$$

where, $i_{g}$ is the greatest gray value of each initial saliency so far.

If a dequeued point holds Equation 4 , its neighbor points will be enqueued if they hold Equation 3. The region-growing process will stop when there is no more points in queue. Figure 2.(d) shows an example of this process.

\section{Experiments, Numerical Results and Discussion}

A database composed of 200 complex postal envelope images, with no fixed position for the handwritten address blocks, postmarks and stamps was used. Each grayscale image, approximately $1500 \times 2200$ pixels, was digitized at $200 \mathrm{dpi}$. We could verify that the address blocks, stamps and postmarks represent only $1.5 \%, 4.0 \%$ and $1.0 \%$ on 


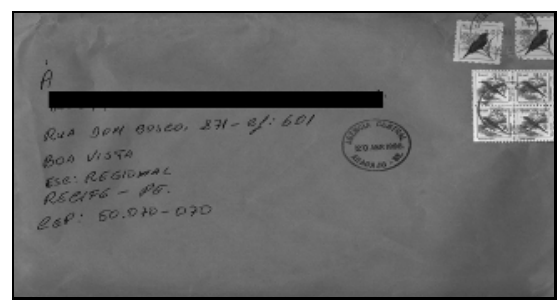

(a)

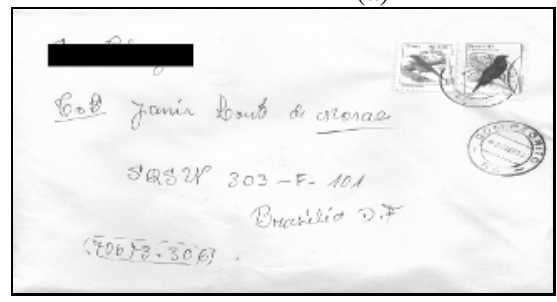

(c)

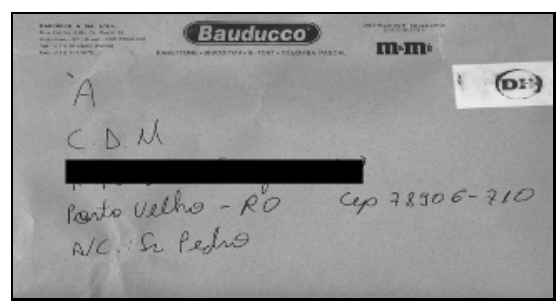

(b)

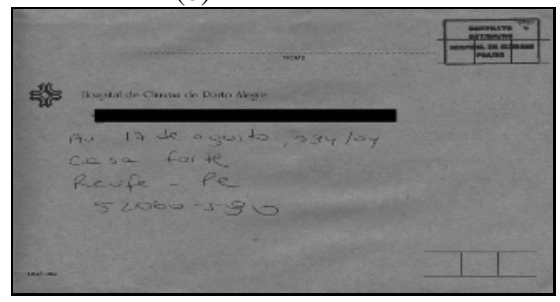

(d)

Fig. 3. Four (4) different images of postal envelopes used, as $I_{i n}$, in the experiments

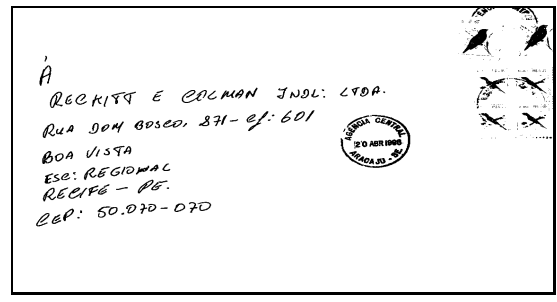

(a)

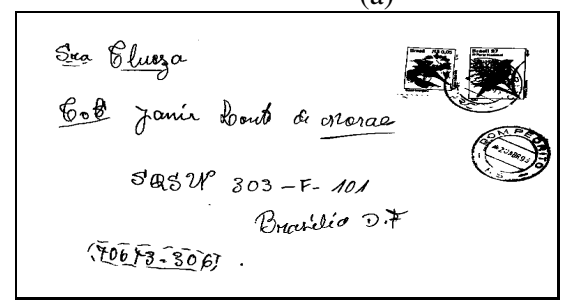

(c)

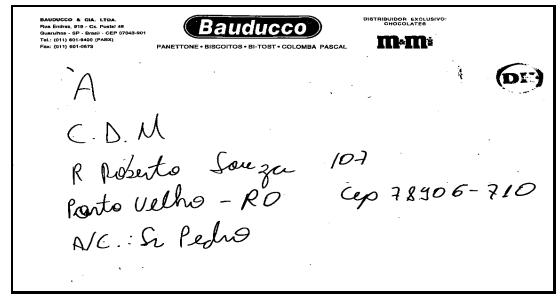

(b)

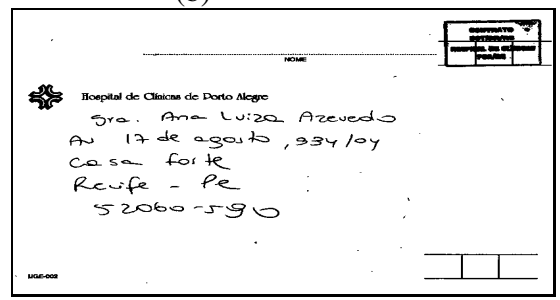

(d)

Fig. 4. Final results $I_{\text {out }}$ obtained by our proposed approach with recovered address block, stamps and postmarks without background, for 4 different envelopes

average of the envelope area, respectively and that the great majority of pixels of these images belong to the envelope background (approximately $93.5 \%$ ). Figure 3 depicts 4 envelopes issued from this database.

A ground-truth strategy was employed to evaluate the accuracy of the proposed approach. The ideal result (ground-truth segmentation) regarding each class (handwritten address block, postmarks and stamps) has been generated for each envelope image. By 
comparing identical pixels at the same location in the ground-truth images and segmented ones, a score of segmentation was computed.

The accuracy evaluation of the proposed method was carried out focusing the attention to address block. The tradeoff between high address block accuracy and low noise rates has been taken into account. Table 1 depicts the best results, where the lacunarity box size $r=3, k=2$, and $\lambda=10 \%$ ( and $Z_{50 \%-\lambda}=1.28$ ).

Table 1. Best results. Average results with identification of regions (pixel by pixel accuracy) for the images tested.

\begin{tabular}{l|l} 
Objects & Accuracy pixel by pixel $(\mu \pm \sigma)$ \\
\hline \hline Address Block & $97.52 \% \pm 5.72 \%$ \\
\hline Stamp & $31.94 \% \pm 15.10 \%$ \\
\hline Postmarks & $88.07 \% \pm 16.79 \%$ \\
\hline Noise & $0.51 \% \pm 0.75 \%$
\end{tabular}

Independently of the layout and background in the input images (Figure 3), one can observe that the segmentation has succeeded in recovering address blocks, postmarks and stamps, and eliminating the background (Figure 4).

In order to quantify their influence in accuracy, experiments to test each step of the proposed approach have been run. Thus, we have focused our attention on automation purposes, and only address block accuracy and noise have been reported. Table 2 depicts variations in results when the box size $r$ changes. By increasing $r$, the address block accuracy decreases and the noise increases. In addition, by increasing $r$, the approach time complexity increases, since it is $O\left(r^{2} n\right)$ in lacunarity feature extraction. From these experiments, one can conclude that $r=3$ is the best box size.

Table 2. Testing Lacunarity varying box size $r$

\begin{tabular}{c|c|c}
\multirow{2}{*}{ Lac } & \multicolumn{2}{|c}{ Accuracy pixel by pixel $(\mu \pm \sigma)$} \\
\cline { 2 - 3 } & Address Block & Noise \\
\hline \hline 3 & $97.52 \% \pm 5.72 \%$ & $0.51 \% \pm 0.75 \%$ \\
\hline 5 & $97.37 \% \pm 5.95 \%$ & $0.52 \% \pm 0.76 \%$ \\
\hline 7 & $97.14 \% \pm 6.42 \%$ & $0.52 \% \pm 0.77 \%$ \\
\hline 9 & $96.80 \% \pm 7.10 \%$ & $0.53 \% \pm 0.76 \%$
\end{tabular}

The influence of multiplicative factor of non-linear normalization has also been tested (Table 3). No meaningful modification in accuracy has occurred. We can conclude that feature extraction and proposed non-linear normalization based on standard deviation are robust.

The influence of parameter $\lambda$ has also been analyzed (Table 4). By observing the results regarding accuracy for address block and noise, one can clearly observe how $\lambda$ 
Table 3. Testing non-linearity factor normalization $k$

\begin{tabular}{c|c|c}
\multirow{2}{*}{ factor } & \multicolumn{2}{|c}{ Accuracy pixel by pixel $(\mu \pm \sigma)$} \\
\cline { 2 - 3 } & Address Block & Noise \\
\hline \hline 0.25 & $97.80 \% \pm 5.35 \%$ & $0.80 \% \pm 1.04 \%$ \\
\hline 0.5 & $97.77 \% \pm 5.37 \%$ & $0.66 \% \pm 0.88 \%$ \\
\hline 1 & $97.71 \% \pm 5.46 \%$ & $0.59 \% \pm 0.82 \%$ \\
\hline 2 & $97.52 \% \pm 5.72 \%$ & $0.51 \% \pm 0.75 \%$ \\
\hline 3 & $97.33 \% \pm 5.97 \%$ & $0.48 \% \pm 0.72 \%$ \\
\hline 4 & $97.13 \% \pm 6.28 \%$ & $0.45 \% \pm 0.72 \%$
\end{tabular}

parameter can affect the accuracy. Increasing (decreasing) $\lambda$ increases (decreases) both address block and noise accuracies. By considering $0.51 \%$ a good noise rate on average, $\lambda=10 \%$ has been chosen.

Table 4. Testing objects image distribution, the $\lambda$ parameter

\begin{tabular}{c|c|c}
\multirow{2}{*}{$\lambda$} & \multicolumn{2}{|c}{ Accuracy pixel by pixel $(\mu \pm \sigma)$} \\
\cline { 2 - 3 } & Address Block & Noise \\
\hline \hline $17 \%$ & $98.56 \% \pm 3.71 \%$ & $0.87 \% \pm 1.21 \%$ \\
\hline $15 \%$ & $98.34 \% \pm 4.10 \%$ & $0.77 \% \pm 1.11 \%$ \\
\hline $10 \%$ & $97.52 \% \pm 5.72 \%$ & $0.51 \% \pm 0.75 \%$ \\
\hline $5 \%$ & $94.77 \% \pm 9.32 \%$ & $0.28 \% \pm 0.45 \%$ \\
\hline $2.5 \%$ & $91.98 \% \pm 12.97 \%$ & $0.17 \% \pm 0.29 \%$
\end{tabular}

These experiments have shown that the accuracy is biased only for $\lambda$ parameter, which is used to apply the knowledge about database images. One can say that the Gaussian supposition works well (global constrain - Equation 3). On other hand, the value choice of the other parameters was not critical in our proposed segmentation method.

\section{Conclusions}

A new postal envelope segmentation method based on saliency identification from lacunarity feature was proposed. The obtained results have shown this approach very promising. The lacunarity algorithm is simple to implement, depending only on local means and variances calculated for window sizes throughout the image. There is no need to correct for noise in the image. Hence, lacunarity by itself may be sufficient to characterize postal envelope texture, address block, postmarks and stamps, resulting in a major advantage over other approaches. The time complexity $(O(n))$ of the regiongrowing algorithm is the same as in other approaches. But, simplicity of this approach gives a time performance gain (6 times faster), compared with [2], which performs an iterative process ( $\mathrm{k}$-means algorithm) and uses large box sizes for computing the fractal dimension. 


\section{Acknowledgments}

We would like to acknowledge support for this research from UFMG, PUCPR, $\mathrm{CNPq} / \mathrm{MCT}, \mathrm{CAPES} / \mathrm{MEC}$ and the Brazilian Post Office Agency (Correios Brasileiros).

\section{References}

1. Wolf, M., Niemann, H., Schmidt, W.: Fast address block location on handwritten and machine printed mail-piece images. In: ICDAR'97 IEEE International Conference on Document Analysis and Recognition, Ulm, Germany (1997) 753-757

2. Eiterer, L., Facon, J., Menoti, D.: Postal envelope address block location by fractal-based approach. SIBGRAPI/SIACG 2004, XVII Brazilian Symposium on Computer Graphics and Image Processing 1 (2004) 90-97

3. Mandelbrot, B.: The Fractal Geometry of Nature. W. H. Freeman And Company, New york (1983)

4. Henebry, G., Kux, H.: Lacunarity as a texture measure for sar imagery. International Journal of Remote Sensing 16 (1995) 565-571

5. Lin, B., Yang, Z.R.: A suggested lacunarity expression for sierpinski carpets. Journal of Physics A: Mathematical and General 19 (1986) 49-52

6. Gefen, Y., Meir, Y., Mandelbrot, B.B., Aharony, A.: Geometric implementation of hypercubic lattices with noninteger dimensionality by use of low lacunarity fractal lattices. Physical Review Letters 50 (1983) 145-148

7. Allain, C., Cloitre, M.: Characterizing the lacunarity of random and deterministic fractal sets. Physical Review A 44 (1991) 3552-3558

8. Otsu, N.: A threshold selection method from gray-level histograms. IEEE Transactions on Systems, Man and Cybernetics 9 (62-66) 1979 